\title{
Traditional or regenerative periodontal surgery?-a comparison of the publications between two periodontal journals over time
}

Staubli, Noémie ; Schmidt, Julia C ; Buset, Sabrina L ; Gutekunst, Claudia J ; Rodriguez, Fabiola R ; Schmidlin, Patrick R ; Walter, Clemens

\begin{abstract}
OBJECTIVES The objective is to compare the amount and content of publications regarding traditional or regenerative periodontal surgery in the years 1982/1983 and 2012/2013 in two leading periodontal journals of North America and Europe. MATERIAL AND METHODS The search was carried out in the Journal of Periodontology and Journal of Clinical Periodontology. Four reviewers screened the articles and allocated the topics with respect to periodontal surgery. The distribution of articles with respect to traditional or regenerative periodontal surgery was then compared between the journals and the respective time periods. RESULTS Out of 1084 screened articles, 145 articles were included. Articles with periodontal surgery content amounted to $18 \%$ for the first time period and to $11 \%$ for the second time period. In the years $1982 / 1983$, 7\% of articles in the Journal of Periodontology and $8 \%$ in the Journal of Clinical Periodontology referred to traditional periodontal surgery, while 8\% (Journal of Periodontology) and 5\% (Journal of Clinical Periodontology) examined regenerative periodontal surgery. The distribution changed 30 years later, with 1\% (Journal of Periodontology) and 3\% (Journal of Clinical Periodontology) traditional periodontal surgery and $7 \%$ and $6 \%$ regenerative periodontal surgery content. CONCLUSION While the clinical need for traditional periodontal surgery remained, research in this important field decreased. Publications rather tended to focus on adjunctive regenerative measures. CLINICAL RELEVANCE Periodontal surgery with adjunctive regenerative measures is an established and well-documented clinical procedure. However, with respect to the dominance of horizontal bone loss in periodontally diseased patients, there is a need for ongoing research with focus on traditional periodontal surgery.
\end{abstract}

DOI: https://doi.org/10.1007/s00784-017-2159-2

Posted at the Zurich Open Repository and Archive, University of Zurich

ZORA URL: https://doi.org/10.5167/uzh-145033

Journal Article

Accepted Version

Originally published at:

Staubli, Noémie; Schmidt, Julia C; Buset, Sabrina L; Gutekunst, Claudia J; Rodriguez, Fabiola R; Schmidlin, Patrick R; Walter, Clemens (2018). Traditional or regenerative periodontal surgery?-a comparison of the publications between two periodontal journals over time. Clinical Oral Investigations, 22(1):29-46.

DOI: https://doi.org/10.1007/s00784-017-2159-2 
Traditional or regenerative periodontal surgery? - A comparison of the publications between two periodontal journals over time

Noémie Staubli ${ }^{1}$, Julia C. Schmidt ${ }^{1}$, Sabrina L. Buset ${ }^{2}$, Claudia J. Gutekunst $^{1}$, Fabiola R. Rodriguez ${ }^{3}$, Patrick R. Schmidlin ${ }^{4}$, Clemens Walter ${ }^{1 *}$

${ }^{1}$ Department of Periodontology, Endodontology and Cariology, University Centre for Dental Medicine, University of Basel, Hebelstrasse 3, 4056 Basel, Switzerland ${ }^{2}$ Department for Reconstructive Dentistry and Temporomandibular Disorders, University Centre for Dental Medicine, University of Basel, Hebelstrasse 3, 4056 Basel, Switzerland

${ }^{3}$ Private dental office Dentilus AG, Forchstrasse 99, 8032 Zurich, Switzerland ${ }^{4}$ Clinic of Preventive Dentistry, Periodontology and Cariology, Centre of Dental Medicine, University of Zurich, Plattenstrasse 11, 8032 Zurich, Switzerland

Running title: periodontal surgery over time

Keywords: periodontal surgery; bibliometrics; periodontal research; journal of periodontology; journal of clinical periodontology

${ }^{*}$ Corresponding author:

Clemens Walter

Department of Periodontology, Endodontology and Cariology, University Centre for Dental Medicine, University of Basel

Hebelstrasse 3

4056 Basel

Switzerland

Phone: +41-61-2672628 
Fax: +41-61-2672659

Email: clemens.walter@unibas.ch 


\section{Abstract}

Objectives To compare the amount and content of publications regarding traditional or regenerative periodontal surgery in the years $1982 / 83$ and $2012 / 13$ in two leading periodontal journals of North America and Europe.

Material and methods The search was carried out in the Journal of Periodontology and Journal of Clinical Periodontology. Four reviewers screened the articles and allocated the topics with respect to periodontal surgery. The distribution of articles with respect to traditional or regenerative periodontal surgery was then compared between the journals and the respective time periods.

Results Out of 1084 screened articles, 145 articles were included. Articles with periodontal surgery content amounted to $\underline{18 \%}$ for the first time period and to $\underline{11 \%}$ for the second time period. In the years 1982/83, in the Journal of Periodontology $7 \%$ and in the Journal of Clinical Periodontology $8 \%$ of articles referred to traditional periodontal surgery, while $8 \%$ (Journal of Periodontology) and 5\% (Journal of Clinical Periodontology) examined regenerative periodontal surgery. The distribution changed 30 years later, with $1 \%$ (Journal of Periodontology) and $3 \%$ (Journal of Clinical Periodontology) traditional periodontal surgery and $\underline{7 \%}$, respectively $6 \%$ regenerative periodontal surgery content.

Conclusion While the clinical need for traditional periodontal surgery remained, research in this important field decreased. Publications rather tended to focus on adjunctive regenerative measures.

Clinical Relevance Periodontal surgery with adjunctive regenerative measures is an established and well documented clinical procedure. However, with respect to the dominance of horizontal bone loss in periodontally diseased patients there is a need for ongoing research with focus on traditional periodontal surgery. 


\section{Introduction}

With the increasing understanding of the pathogenesis of periodontal diseases, the approaches of periodontal therapy changed. A virtual origin of modern periodontology dates back to the beginning of the 20th century with the description of the surgical treatment of pyorrhea alveolaris, including the elimination of the "infected" alveolar bone [1]. Later on, the infection was localized in the gingival tissues, leading to the gingivectomy approach [2]. Any complications caused by this procedure raised the development of techniques aiming in a preservation or transplantation of the attached gingiva such as apically repositioned flap or free gingival grafts [3-5].

Around the year 1980, first attempts were made to evaluate the non-surgical and surgical periodontal therapies by direct comparisons. While the outcomes were comparable, more gingival recessions were observed with surgical periodontal therapies [6, 7]. According to this seminal research, a "cut-off" for a distinctive periodontal pocket depth (PPD) was defined for decision-making between nonsurgical treatment and traditional periodontal surgery [8]. Even in the early 80's a novel innovative - the so-called - "regenerative approach" came across and gained increasing attendance [9-12]. These publications described research on an additional treatment of the exposed root surface and/or bony defect aiming in enhancing the regeneration of the tooth supporting structures.

While the indications for periodontal surgery seem to decrease over time, at least two opposed surgical options - a traditional and a regenerative approach - were currently discussed for teeth in need for further surgical treatment [13]. Most of the research on the development of periodontal therapy was published in the "North American" Journal of Periodontology (JP), founded in 1930 by the American Academy of Periodontology (AAP, first edition dated January 1930, [14]), and in the 
"European" Journal of Clinical Periodontology (JCP), founded in 1974 by Jan Lindhe (JCP, first edition dated March 1974, [15]). These journals may represent at least to some extent the North American and the European school of periodontology and their academic societies.

Recently, the prospective trends in periodontal research were discussed within the DELPHI-Project [16]. Using questionnaires distributed to public health providers and/or dental practioners in a private office or university setting, a consensus on current developments and expectations was published. In addition to this personalized questionnaire approach, it may be helpful to analyze retrospectively the trends in periodontal publishing in certain research areas to identify future needs for clinically relevant research activities.

The aim of this bibliometric study was to explore the possible changes in reporting on periodontal surgery over the last three decades in the Journal of Periodontology and Journal of Clinical Periodontology.

\section{Materials and Methods}

Focused questions

a) How many publications dealing with periodontal surgery were published in the Journal of Periodontology (JP) and Journal of Clinical Periodontology (JCP) in the years 1982/83 and 2012/13?

b) Was there a change regarding the content of publications focusing on traditional or regenerative surgery over time?

c) With respect to articles with traditional or regenerative periodontal surgery content, was there a difference between these two periodontal journals? 
Selected journals and timeframe

The search was carried out in the two currently leading highly ranked peer-reviewed periodontal journals with different geographical origin representing the academic societies of North America and Europe. The JP was ranked within ISI (Institute for Scientific Information) Journal Citation Report (JCR) on position 11 with an impact factor (IF) 2.844 in 2015 . The corresponding data for the JCP were 3.915 (IF) and position 6 in 2015 [17]. All articles published in these journals in the years 1982/83 and 2012/13 were analyzed.

\section{Classification of the articles}

Three tree diagrams (TD-A, TD-B and TD-C) were developed for classification of articles (Appendix 1-3).

In TD-A, the articles were classified in terms of study design. In level 1 , the articles were analyzed for their main methodology and hierarchically coded (Appendix 1). The classification of study designs considered: "original study", "review", "case report, case series", "comment, letter, editorial, erratum" and "others". In level 2, the variable "original study" was further divided in "clinical study", "in vitro study" and "animal study". Finally, in level 3, a "clinical study" was classified as "intervention study" (e.g. randomized controlled trial) or "epidemiological study". The allocations were assigned to the coding scheme within the TD (Appendix 1).

TD-B and TD-C considered the articles in terms of their content. The articles were analyzed for their main content and hierarchically coded (Appendix 2-3). The two main contents were: "periodontal conditions and therapy" (TD-B) and "periimplant conditions and therapy" (TD-C). In level 1, these groups were further subdivided in "anatomy", "etiology and pathogenesis", "diagnostics", "therapy", "quality of life" and "medicine". The main content "peri-implant conditions and 
therapy" also included "implant characteristics" and "implant installation and socket/ridge preservation". Articles not assigned to these groups were categorized as "others". The content "periodontal therapy" was divided in "accompanying therapy (e.g. tobacco use cessation, oral hygiene instruction, motivational interviewing)", "non-surgical therapy", "periodontal surgery", "supportive periodontal therapy, prognosis and risk assessment", "interdisciplinary therapy" and "others" (level 2). In level 3, "periodontal surgery" was divided in "traditional", "regenerative" and "mucogingival therapy" (please see below for further descriptions). Finally, articles were assigned to groups (level 4) such as "surgical approach", "adjunctive materials" and "pharmacological protocol". The latter group was subdivided in "local" or "systemic application" (level 5) both further divided in "antibiotics" and "others" (e.g. disinfectants for local and drugs for systemic application) (level 6).

For TD and/or level within TD, an article could be assigned to different groups. For example, a publication describing a (virtual) clinical study comparing the outcomes of traditional versus regenerative periodontal surgery using membranes in furcation-involved molars was allocated in level 4 to the contents: "surgical approach" in "traditional therapy" and "surgical approach" in "regenerative therapy".

\section{Evaluation of publications}

After a calibration procedure, including a detailed description of the study design and a test evaluation, four authors (J. S., S. B., F. R., N. S.) were involved in the evaluation process.

A hand search of the two selected journals (JP and JCP) and the two specific time periods (1982/83 and 2012/13) was performed. Each title and abstract and additionally the full texts of questionable publications were screened independently by two authors according to the TDs (Appendix 1-3). When conflicting results were 
found regarding the allocation of an article, an independent and blinded reassessment was conducted by the two other authors. Dissenting allocations of the second assessment compared to the first assessment regarding the classification of the surgical content were discussed between the authors and the principal investigator (C. W.). Every author was involved in both screening processes.

The following variables were recorded for each article and included in the analysis: Year and month of publication, author(-s), title, journal, main study design and content(-s) (Tables $1 \mathrm{a}, \mathrm{b}$ and $2 \mathrm{a}, \mathrm{b})$.

Inclusion criteria and applied definition:

"Clinical studies", "animal stadies", "reviews", "case reports and case series" with periodontal surgery content were considered in this analysis. The included articles focused on surgical approaches, adjunctive materials and/or pharmacological protocols.

Articles classified as traditional periodontal surgery considered publications within the following field of research: Open flap debridement techniques applied without distinctive materials aiming in enhancing the regeneration of the periodontium.

Articles classified as regenerative periodontal surgery considered publications within the following field of research: Open flap debridement surgery with additional treatment of the exposed root surface and/or bony defect aiming in enhancing the regeneration of the tooth supporting structures.

Articles classified as mucogingival periodontal surgery considered publications within the following field of research: Treatment of mucogingival defects according to the classification of periodontal diseases [18].

In a further description of the entire studies any supplemental surgical materials 
and/or pharmacological protocols were, if necessary, considered.

Exclusion criteria and applied definition:

"In vitro studies", "comments, letters, editorials, erratums" and "others" were excluded. Excluded articles considered exclusively contents such as anatomy, etiology and pathogenesis, diagnostics, quality of life, periodontal/peri-implantitis medicine, implant characteristics and implant placement not in conjunction with traditional, regenerative or mucogingival periodontal surgery.

\section{Synthesis of results and analysis}

The data were descriptively presented and a frequency distribution of the journals content in the time periods $1982 / 83$ and $2012 / 13$ was shown [19]. According to the number of involved reviewers and the possibility of classifying an article to more than one category, the number of articles sums up to more than the real number of articles. The agreement between the reviewers within the respective TD was calculated and presented as percentage value of agreement.

\section{Results}

The initial agreement between the reviewers within the respective TD was $94.5 \%$ for the study design (TD-A) and $83.3 \%$ for its content (TD-B).

The data were presented according to a modification of the PICO - approach, i.e. population/participants $(P)$, intervention $(I)$, comparison $(C)$, outcome $(O)-[20]$ : (P) - Number of screened articles and authors

A total of 1084 articles were screened. In JP, 208 articles were published in the period 1982/83 and 442 thirty years later, i.e. 2012/13 (Figures 1a,b). The corresponding numbers for JCP accounted for 118 and 316, respectively (Figures 
2a,b). Out of these 1084 articles, 145 articles met the inclusion criteria (Tables 1a,b and $2 a, b)$.

With respect to TD-A ("study design"), 72 articles were defined as an intervention study, 7 articles as an epidemiological study, 36 articles as an animal study, 11 articles as a review and 19 articles as a case report/case series.

Regarding the classification in terms of periodontal surgery content within TDB ("periodontal conditions and therapy"), 39 articles were classified as traditional periodontal surgery, $\underline{73}$ articles as regenerative periodontal surgery and $\underline{38}$ articles as mucogingival periodontal surgery (Figures $1 a, b$ and $2 a, b)$. The number of the 150 allocations exceeded the number of the 145 included publications. Four publications were allocated to more than one periodontal surgery theme, i.e. to traditional and/or regenerative and/or mucogingival periodontal surgery.

The numbers of authors in each article depended on the year of publication and journal. A mean of 2.9 authors (JP) respectively 2.6 authors (JCP) contributed in an average article in 1982/83. In contrast, 5.2 authors (JP) and $\underline{6.6}$ authors (JCP) coauthored an average article thirty years later.

(I/C) Content of screened articles

Articles classified as traditional periodontal surgery considered surgical techniques such as modified Widman flap, apically positioned flap and mucoperiostal flap with an additional open flap debridement. The surgical intervention included hemisection, trisection, root amputation and/or pre-molarization.

The surgical intervention in articles distributed to regenerative periodontal surgery included adjunctive materials such as dentin matrix gelatine, enamel matrix proteins, mesenchymal stem cells, porous titanium granules, acellular dermal matrix grafts, autologous, allogenic, xenogenous or alloplastic bone grafts, demineralized 
dentin grafts, nonresorbable ceramic (durapatite) grafts, growth factors, platelet concentrates (autologous platelet-rich fibrin, platelet-rich plasma) and membranes (e.g. biodegradable collagen membrane) and/or the application of citric, sodium deoxycholate or phosphoric acids. For example, suture materials and videoscopes were considered as adjunctive materials.

Pharmacological protocols in articles distributed to traditional, regenerative and mucogingival periodontal surgery contained the prescription of systemic antibiotics (e.g. cephalexin, amoxicillin and metronidazole) and locally administered antibiotics (e.g. doxycycline, tetracycline and minocycline). Local agents without allocation to the group of antibiotics included for instance disinfectants (e.g. chlorhexidine) and/or bisphosphonates (e.g. alendronate). Systemic agents other than antibiotics comprised for example bisphosphonates (e.g. alendronate) and analgetics (e.g. paracetamol). Local chemical protocols belonging to the field of research regenerative periodontal surgery comprised also antibiotic-containing membranes (e.g. doxycycline-loaded biodegradable membrane) and/or demineralisation of denuded root surfaces with citric, sodium deoxycholate or phosphoric acids.

As outcome measures in traditional, regenerative and mucogingival periodontal surgery served histologic parameters (e.g. laminin 5 and Type IV collagen expressions on immunohistochemically stained samples for evaluation of new epithelial formation), adverse effects (e.g. caused by smoking), discomfort (e.g. questionnaire, visual analogue scale, oral health related quality of life), clinical parameters (e.g. PPD reductions, furcation involvement, gain of clinical attachment, dimension of gingival recession or keratinized tissue, measures of oral hygiene or gingival inflammation), radiographic parameters (radiographic changes of the osseous defect) and/or survival of the tooth. 
(O) Outcome

a) How many publications dealing with periodontal surgery were published in the Journal of Periodontology (JP) and Journal of Clinical Periodontology (JCP) in the years 1982/83 and 2012/13?

Articles with periodontal surgery content accounted for 38 articles (18\%) in JP and 20 articles (17\%) in JCP for the first time period and 49 articles $(11 \%)$ in JP and 38 articles $(12 \%)$ in JCP for the second time period, 30 years later. The value in percentage describes the proportion of the articles with periodontal surgery content out of all published articles in the corresponding journal and time period.

\section{b) Was there a change regarding the content of publications focusing on} traditional or regenerative surgery over time?

The total number of articles published in JP and JCP increased from 326 to 758 articles over time. Out of all publications in 1982/83, 24 articles (7\%) investigated traditional periodontal surgery, 22 articles $(7 \%)$ regenerative periodontal surgery and 14 articles (4\%) mucogingival periodontal surgery. Thirty years later, the distribution for the three periodontal surgical treatments changed. In 2012/13, articles with traditional periodontal surgery content amounted to $\underline{15}$ articles $(2 \%)$, articles with regenerative periodontal surgery content amounted to 51 articles (7\%) and articles with mucogingival periodontal surgery content amounted to $\underline{24}$ articles (3\%).

Similar to the increased number of total published articles in JP and JCP over time, the absolute number of articles investigating regenerative periodontal surgery increased and the percentage number remained stable, but the absolute and percentage number of articles with traditional periodontal surgery content decreased from $1982 / 83$ to $2012 / 13$. 


\section{c) With respect to articles with traditional or regenerative periodontal surgery}

content, was there a difference between these two periodontal journals?

In 1982/83, JP published a higher absolute number of articles with traditional (15 articles) and regenerative periodontal surgery (16 articles) content compared to JCP ( 9 and 6 articles, respectively). The percentage of articles dealing with traditional (7\% for $\mathrm{JP}$ and $8 \%$ for JCP) and regenerative ( $8 \%$ for JP and $5 \%$ for JCP) periodontal surgery showed an inverse tendency slightly favoring publications with traditional periodontal surgery in JCP and publications with regenerative periodontal surgery in JP. In 2012/13 a higher number of articles reporting on regenerative periodontal surgery was published in JP (31 articles) as compared to JCP (20 articles). JCP showed a higher number with respect to articles on traditional periodontal surgery (10 articles in JCP vs. $\underline{5}$ articles in JP). Both journals showed a similar trend with regard to articles with traditional periodontal surgery over time with a reduction of percentages. The percentage of articles assessing regenerative periodontal surgery remained stable over time with an increasing absolute number, which was linear to the absolute number of all published articles in both journals.

\section{Discussion}

The present analysis demonstrated that JCP and JP followed a similar course over time with a focus on reporting on regenerative periodontal surgery and a diminishment in traditional periodontal surgery. The overall number of publications has more than doubled in the evaluated thirty year period, whereas the total number of studies with periodontal surgery content has not increased accordingly. The analysis showed that more authors have contributed to a single publication in 2012/13. 
The results of this study reveal how the focus changed in research on periodontal surgery from $1982 / 83$ to $2012 / 13$. The years $1982 / 83$ were chosen in order to cover a relevant time frame of 30 years and to tribute the classical publication on guided tissue regeneration (GTR) by Nyman S [21]. At the latest, since the 80 's the innovative regenerative approach was implemented and dramatically influenced the concept of periodontal therapy $[9-12,21]$. The distribution of articles with regenerative versus traditional periodontal surgery content was dominated by publications with the latter content in 1982/83 (Figures 1a,b and 2a,b).

Even though the two periodontal journals showed a similar distribution of articles with periodontal surgical content, they may have been influenced by different renowned researchers and may therefore represent different philosophies. Authors of landmarking articles published in the 70's and 80's in the European JCP were for example Bengt Rosling, Per Axelsson and Jan Lindhe, just to mention a few of them. For example, the studies of Rosling et al. (1976) and Axelsson \& Lindhe (1981) stressed the importance of plaque control and periodontal maintenance $[22,23]$. This approach may reflect a kind of "European school" with enhancing oral hygiene to establish favourable periodontal conditions. Raul Caffesse, Wayne B. Kaldahl, Sigurd Ramfjord and Saul Schluger may be mentioned among outstanding North American representatives of periodontal research of their time. They contributed significantly to periodontal research by publishing new surgical concepts, including osseous surgery $[6,24-26]$. However, the current study was not designed to assess the profiles of JP and JCP and their choice of publications with specific periodontal therapy approaches. As evidenced by several publications, North American authors published in JCP and vice versa $[27,28]$. One can only speculate that the two journals may differ to some extent regarding their authors representing different periodontal approaches. 
Past and future trends and/or expectations may be evaluated by different settings including questionnaires, consensus conferences and/or bibliometric research [16]. Bibliometric research focuses on a descriptive analysis of publications and received increasing attention in recent years [29, 30]. Different aspects relevant for understanding research developments in the past, future trends, geographic origin of the authors, study design and quality, number and nature of measured outcomes, factors influencing diagnosis or the most cited articles in periodontology were the objectives of recent bibliometric studies [29-32].

Our analysis demonstrates that regenerative periodontal surgery became an established procedure and dominates now the literature on periodontal surgery. However, the percentage number of articles with periodontal surgery content decreased over time. In the same time the number of publications focusing on dental implants consistently increased during 1995 and 2010 [32] even in periodontal journals. The above mentioned study by Madianos et al. (2016) found, that the vast majority of experts (95\%) still expect a continuing increase in publications on dental implants. Our study shows that the focus changed to regenerative periodontal surgery (Figures 1a,b and 2a,b). However, most of the defects in need for periodontal surgery are characterized by horizontal bone loss and/or furcation involvement [3336]. In contrast to the external evidence showing the benefits of regenerative measures in vertical bony defects, sufficient evidence showing clinical relevant benefits in furcation and/or horizontal bony defects is still scarce [37, 38]. In addition, the prevalence of teeth with vertical bony defects seems to decrease according to a study in a population of dentally aware individuals [39]. Despite the limited indications for regenerative periodontal surgery the dominance of publications with regenerative surgery content might influence decision-making, maybe leading to treatment approaches not sufficiently supported by external evidence. Recently, in a 
questionnaire survey with 340 respondents a relevant number of participants suggested periodontal surgery with adjunctive regenerative measures for a maxillary molar with through and through furcation involvement [40].

The overall number of publications in JP and JCP has more than doubled within the last 30 years. These results indicate that there is an enormous growth of data. In 1979, about 14 reports of trials were published per day, compared to that, 30 years later, 75 trials and 11 systematic reviews were published per day and the plateau has not reached [41]. These observations lead to the question how to manage these data. Furthermore bibliometric studies may contribute to necessary data management by identifying gaps and asking questions for future dental needs.

As a shortcoming of this study, the results are limited to a retrospective analysis of publications with periodontal surgery within a specific time period of two selected journals. Since another time frame or other journals may potentially reveal varying results, some bias with respect to the interpretation of the data obtained in this study and the conclusions should be kept in mind. However, as a strength of the current investigation, one can argue that the evaluation was independent, blinded and double-controlled by five authors.

\section{Conclusion}

Within the limitations of this study, it might be concluded that the amount of articles with regenerative periodontal surgery content seems to increase whereas articles with traditional periodontal surgery content decreased over time. The journals JP and JCP showed similar trends over time. However, according to clinical reality there is still a need for traditional periodontal surgery. As a trend, publications in recent years focused more on periodontal surgery with adjunctive regenerative measures. 


\section{Compliance with Ethical Standards}

Conflict of interest: Noémie Staubli declares that she has no conflict of interest. Julia C. Schmidt declares that she has no conflict of interest. Sabrina L. Buset declares that she has no conflict of interest. Claudia J. Gutekunst declares that she has no conflict of interest. Fabiola R. Rodriguez declares that she has no conflict of interest. Patrick R. Schmidlin declares that he has no conflict of interest. Clemens Walter declares that he has no conflict of interest.

Funding: The work was supported by the Department of Periodontology, Endodontology and Cariology, University Centre for Dental Medicine, University of Basel, Switzerland.

Ethical approval: This article does not contain any studies with human participants or animals performed by any of the authors.

Informed consent: For this type of study, formal consent is not required. 


\section{References}

1. Neumann R (1912) Die Alveolarpyorrhoe und ihre Behandlung. Meusser, Berlin

2. Kronfeld RJ (1935) Dentition of the bone tissue of the alveolar process below the periodontal pockets. J Periodontol 5:22-29

3. Nabers CL (1954) Repositioning the attached gingiva. J Periodontol 25:38-39

4. Björn H (1963) Free transplantation of gingiva propria. Sver Tandlakarforb Tidn 22:684

5. Nabers JM (1966) Free gingival grafts. Periodontics 4:243-245

6. Ramfjord SP, Knowles JW, Nissle RR, Burgett FG and Shick RA (1975) Results following three modalities of periodontal therapy. J Periodontol $46: 522-526$

7. Isidor F and Karring T (1986) Long-term effect of surgical and non-surgical periodontal treatment. A 5-year clinical study. J Periodontal Res 21:462-472

8. Lindhe J, Socransky SS, Nyman S, Haffajee A and Westfelt E (1982) "Critical probing depths" in periodontal therapy. J Clin Periodontol 9:323-236

9. Lindskog S, Hammerström L (1981) Formation of intermediate cementum. I-III. J Craniofac Genet Dev Biol 2:147-177

10. Karring T, Isidor F, Nyman S and Lindhe J (1984) New attachment formation on citric acid and non-citric acid treated roots. J Periodontal Res 19:666-669

11. Nyman S, Ericsson I, Runstad L and Karring T (1984) The significance of alveolar bone in periodontal disease. An experimental study in the dog. $\mathrm{J}$ Periodontal Res 19:520-525

12. Miron RJ, Sculean A, Cochran DL, Froum S, Zucchelli G, Nemcovsky C, Donos N, Lyngstadaas SP, Deschner J, Dard M, Stavropoulos A, Zhang Y, Trombelli L, Kasaj A, Shirakata Y, Cortellini P, Tonetti M, Rasperini G, Jepsen 
S and Bosshardt DD (2016) Twenty years of enamel matrix derivative: the past, the present and the future. J Clin Periodontol 43:668-683

13. Walter C, Weiger R and Zitzmann NU (2011) Periodontal surgery in furcationinvolved maxillary molars revisited--an introduction of guidelines for comprehensive treatment. Clin Oral Investig 15:9-20

14. American Academy of Periodontology. Timeline of AAP Events: 1906 to 2008. https://www.perio.org/about/100Anniversary. Accessed 13 July 2016

15. Lindhe J and Svanberg G (1974) Influence of Trauma from Occlusion on Progression of Experimental Periodontitis in Beagle Dog. J Clin Periodontol $1: 3-14$

16. Madianos P, Papaioannou W, Herrera D, Sanz M, Baeumer A, Bogren A, Bouchard P, Chomyszyn-Gajewska M, Demirel K, Gaspersic R, Giurgiu M, Graziani F, Jepsen K, Jepsen S, T OB, Polyzois I, Preshaw PM, Rakic M, Reners M, Rincic N, Stavropoulos A, Sutcu S, Verner C and Llodra JC (2016) EFP Delphi study on the trends in Periodontology and Periodontics in Europe for the year 2025. J Clin Periodontol 43:472-481

17. JCR Science Edition (2015) https://www.google.ch/url?sa=t\&rct=j\&q=\&esrc=s\&source=web\&cd=1\&ved=0a hUKEwiamaPS-

dXRAhWrAsAKHQ2ICl8QFggaMAA\&url=https\%3A\%2F\%2Flib.hku.hk\%2Fsite s\%2Fall\%2Ffiles\%2Ffiles\%2Fdenlib\%2Fimpact\%2520factor\%25202015.pdf\& usg=AFQjCNF1ow8h7gTanLNhz53Icx5R5nf4MQ. Accessed 22 May 2016

18. Armitage GC (1999) Development of a classification system for periodontal diseases and conditions. Ann Periodontol 4:1-6 
19. Park JB, Ko Y and Park YG (2015) Letters to the editor: Re: Bibliometrics study on authorship trends in periodontal literature from 1995 to 2010.

Geminiani A, Ercoli C, Feng C, Caton JG. (J Periodontol 2014; 85:e136-e143).

J Periodontol 86:7

20. Miller SA and Forrest JL (2001) Enhancing your practice through evidencebased decision making: PICO, learning how to ask good questions. J Evid Based Dent Pract 1:136-141

21. Nyman S, Lindhe J, Karring T and Rylander H (1982) New attachment following surgical treatment of human periodontal disease. J Clin Periodontol 9: $290-296$

22. Rosling B, Nyman S and Lindhe J (1976) The effect of systematic plaque control on bone regeneration in infrabony pockets. J Clin Periodontol 3:38-53

23. Axelsson $\mathrm{P}$ and Lindhe $\mathrm{J}$ (1981) The significance of maintenance care in the treatment of periodontal disease. J Clin Periodontol 8:281-294

24. Schluger S (1949) Osseous resection; a basic principle in periodontal surgery. Oral Surg Oral Med Oral Pathol 2:316-325

25. Allen DR and Caffesse RG (1983) Comparison of results following modified Widman flap surgery with and without surgical dressing. J Periodontol 54:470475

26. Kaldahl WB, Kalkwarf KL, Patil KD, Dyer JK and Bates RE, Jr. (1988) Evaluation of four modalities of periodontal therapy. Mean probing depth, probing attachment level and recession changes. J Periodontol 59:783-793

27. Caffesse RG, Sweeney PL and Smith BA (1986) Scaling and root planing with and without periodontal flap surgery. J Clin Periodontol 13:205-210

28. Ericsson I and Lindhe $\mathrm{J}$ (1984) Lack of significance of increased tooth mobility in experimental periodontitis. J Periodontol 55:447-452 
29. Fleming PS, Koletsi D, O'Brien K, Tsichlaki A and Pandis N (2016) Are dental researchers asking patient-important questions? A scoping review. J Dent $49: 9-13$

30. Leow NM, Hussain Z, Petrie A, Donos N and Needleman IG (2016) Has the quality of reporting in periodontology changed in 14 years? A systematic review. J Clin Periodontol 43:833-838

31. Nieri M, Saletta D, Guidi L, Buti J, Franceschi D, Mauro S and Pini-Prato G (2007) Citation classics in periodontology: a controlled study. J Clin Periodontol 34:349-358

32. Geminiani A, Ercoli C, Feng C and Caton JG (2014) Bibliometrics study on authorship trends in periodontal literature from 1995 to 2010. J Periodontol 85:e136-143

33. Nordland P, Garrett S, Kiger R, Vanooteghem R, Hutchens LH and Egelberg J (1987) The effect of plaque control and root debridement in molar teeth. J Clin Periodontol 14:231-136

34. Loos B, Nylund K, Claffey N and Egelberg J (1989) Clinical effects of root debridement in molar and non-molar teeth. A 2-year follow-up. J Clin Periodontol 16:498-504

35. Papapanou PN and Wennstrom JL (1991) The Angular Bony Defect as Indicator of Further Alveolar Bone Loss. J Clin Periodontol 18:317-322

36. Sachs M, Ehmke B (2013) Resektive Knochenchirurgie in der Parodontologie. Eine bewährte Therapieform. Parodontol 24:49-65

37. Jepsen S, Eberhard J, Herrera D and Needleman I (2002) A systematic review of guided tissue regeneration for periodontal furcation defects. What is the effect of guided tissue regeneration compared with surgical debridement in the 
treatment of furcation defects? J Clin Periodontol 29 Suppl 3:103-16;

discussion 160-2

38. Di Tullio M, Femminella B, Pilloni A, Romano L, D'Arcangelo C, De Ninis $P$ and Paolantonio M (2013) Treatment of supra-alveolar-type defects by a simplified papilla preservation technique for access flap surgery with or without enamel matrix proteins. J Periodontol 84:1100-1110

39. Baljoon M, Natto S and Bergstrom J (2003) Occurrence of vertical bone defects in dentally aware individuals. Acta Odontol Scand 61:47-51

40.Zitzmann NU, Scherrer SS, Weiger R, Lang NP and Walter C (2011) Preferences of dental care providers in maintaining compromised teeth in relation to their professional status: implants instead of periodontally involved maxillary molars? Clin Oral Implants Res 22:143-50

41. Bastian H, Glasziou P and Chalmers I (2010) Seventy-five trials and eleven systematic reviews a day: how will we ever keep up? PLoS Med 7:e1000326 
Supporting information captions

\section{Table legend}

Table 1a: Included articles published in the JP in the years 1982/83 with the allocated theme(s) and study design.

Table 1b: Included articles published in the JP in the years 2012/13 with the allocated theme(s) and study design.

Table 2a: Included articles published in the JCP in the years 1982/83 with the allocated theme(s) and study design.

Table 2b: Included articles published in the JCP in the years 2012/13 with the allocated theme(s) and study design. 
Figure legend

\section{JP $1982 / 83(n=208)$}

$15(7 \%)$

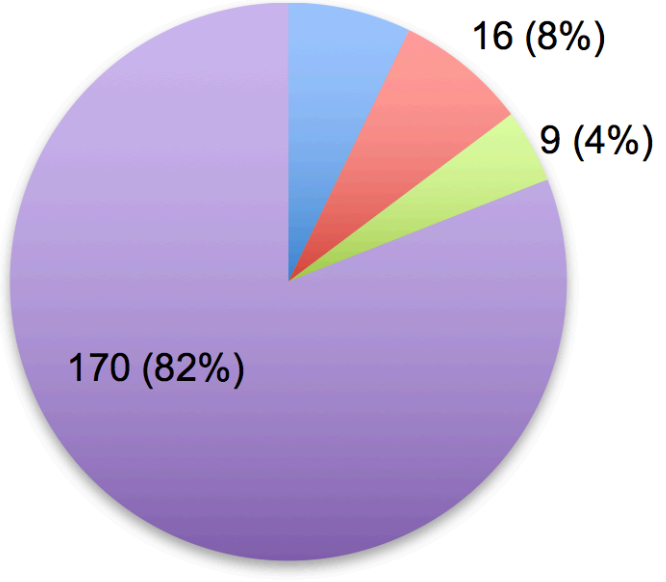

- Traditional periodontal surgery

Regenerative periodontal surgery

Mucogingival surgery

Others

Figure 1a: Chart represents the proportion of articles with traditional, regenerative and mucogingival periodontal surgery content out of a total amount of 208 published articles in the JP in the years $1982 / 83$. Two publications were allocated to traditional and regenerative periodontal surgery, i.e. were distributed to both groups.

\section{JP 2012/13 (n=442)}

$5(1 \%) \quad 31(7 \%)$

$14(3 \%)$

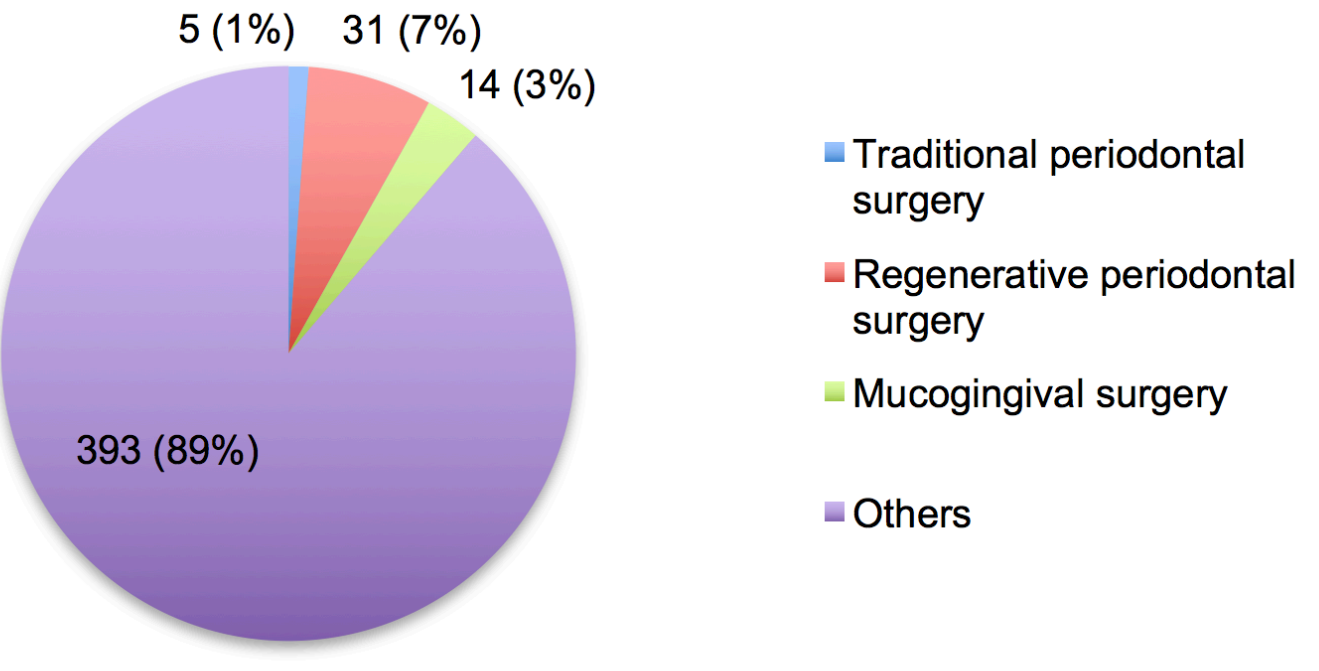


Figure 1b: Chart represents the proportion of articles with traditional, regenerative and mucogingival periodontal surgery content out of a total amount of 442 published articles in the JP in the years $2012 / 13$. One publication was allocated to regenerative and mucogingival periodontal surgery, i.e. was distributed to both groups.

\section{JCP 1982/83 ( $n=118)$}

$9(8 \%)$

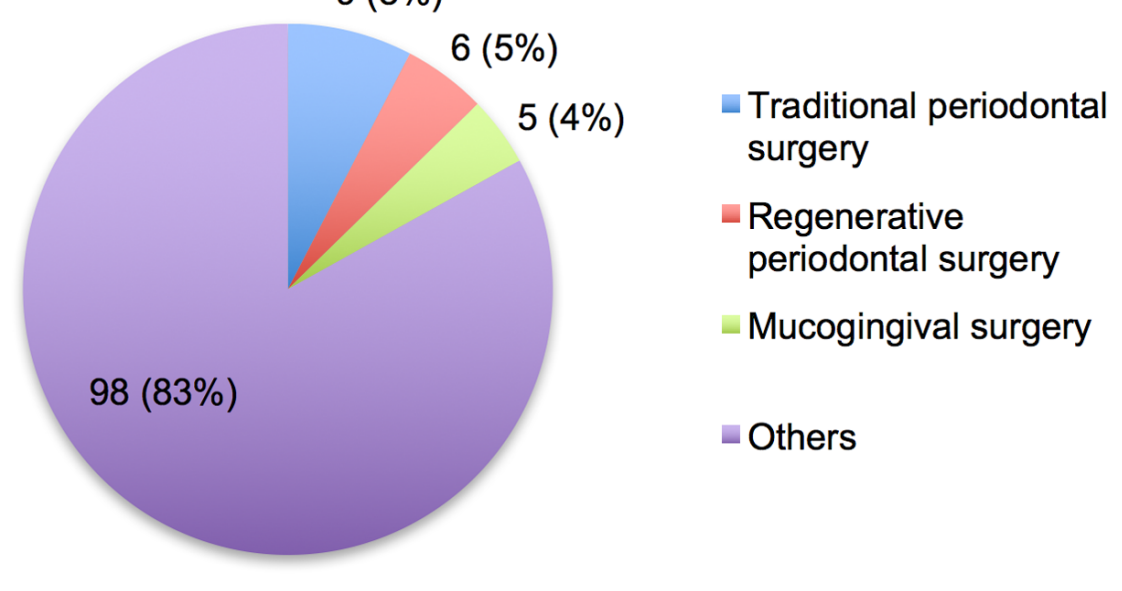

Figure 2a: Chart represents the proportion of articles with traditional, regenerative and mucogingival periodontal surgery content out of a total amount of 118 published articles in the JCP in the years 1982/83. 


\section{JCP 2012/13 (n=316)}

$10(3 \%) 20(6 \%)$

$10(3 \%)$

\section{$278(88 \%)$}

- Traditional periodontal surgery

Regenerative periodontal surgery

Mucogingival surgery

Others

Figure 2b: Chart represents the proportion of articles with traditional, regenerative and mucogingival periodontal surgery content out of a total amount of 316 published articles in the JCP in the years $2012 / 13$. One publication was allocated to traditional, regenerative and mucogingival periodontal surgery, i.e. was distributed to three groups.

\section{Appendix legend}

Appendix 1: Tree diagram A (TD-A) for study design allocation for the screened publications.

Appendix 2: Tree diagram B (TD-B) for theme allocation for publications with periodontal conditions and therapy content.

Appendix 3: Tree diagram C (TD-C) for theme allocation for publications with periimplant conditions and therapy content. 